Voix et Images

voixetimages

\title{
Gauvreau et la critique baroque
}

\section{André G. Bourassa}

Volume 3, numéro 1, septembre 1977

Nicole Brossard

URI : https://id.erudit.org/iderudit/200085ar

DOI : https://doi.org/10.7202/200085ar

Aller au sommaire du numéro

Éditeur(s)

Les Presses de l'Université du Québec

ISSN

0318-9201 (imprimé)

1705-933X (numérique)

Découvrir la revue

Citer cet article

Bourassa, A. G. (1977). Gauvreau et la critique baroque. Voix et Images, 3(1),

19-31. https://doi.org/10.7202/200085ar

Ce document est protégé par la loi sur le droit d'auteur. L'utilisation des services d'Érudit (y compris la reproduction) est assujettie à sa politique d'utilisation que vous pouvez consulter en ligne.

https://apropos.erudit.org/fr/usagers/politique-dutilisation/
Cet article est diffusé et préservé par Érudit.

Érudit est un consortium interuniversitaire sans but lucratif composé de l’Université de Montréal, l'Université Laval et l'Université du Québec à Montréal. Il a pour mission la promotion et la valorisation de la recherche. https://www.erudit.org/fr/ 


\title{
Gauvreau et la critique baroque ${ }^{1}$
}

\author{
IVULKA - Bah! tu peux toujours écrire des \\ phrases de poésie éthérée si tu t'en sens capable \\ ... ça c'est inoffensif et ça peut servir de théra- \\ peutique. Mais plus une syllabe de critique ${ }^{2}$.
}

On parle souvent de Claude Gauvreau en tant que poète et dramaturge. La parution, en juin dernier, d'Étal mixte ${ }^{3}$ et la récente publication des $\mathcal{E}$ Euvres créatrices complètes ${ }^{4}$ va sûrement faire croître la connaissance de cette œuvre que des mises en scène diverses et des films ont aidé à mettre en lumière.

Or la critique de Gauvreau est, comme son œuvre créatrice, d'une qualité exceptionnelle. A la fois violente et savante, elle allie les contraintes en une phrase baroque qu'il se plaît à identifier comme telle parce que le baroque des automatistes lui plaît en critique comme en art et en poésie 5 . L'actualité et la diversité de cette critique dont le texte est en partie inaccessible parce qu'inédit méritent qu'on s'y arrête. Ne serait-ce que pour comprendre le sens ici du mot "baroque".

Les textes critiques de Gauvreau se divisent en trois grandes séries. II y a les comptes rendus, particulièrement ceux qui ont paru dans la chronique «Masques et bergamasques» du journal le Haut-parleur. II y a les grands textes polémiques, comme ceux qu'ont publiés le Quartier latin, Combat, Situations, la Revue socialiste et Liberté. II y a enfin les essais, lettres et conférences comme l'Épopée automatiste vue par un cyclope, Dix-sept lettres à un fantôme et Débat sur la peinture des automatistes. Ces instances critiques seront abordées ici par catégories.

\section{Le chroniqueur}

Gauvreau tint la chronique artistique et littéraire du Haut-parleur durant un an et demi. II couvrit la saison qui commençait avant l'automne de 1950 (30 juillet) pour se rendre à la fin de l'automne de 1951 (22 décembre). Les expériences qui précédèrent son entrée au journal de $T$. Damien Bou- 
chard sont relativement nombreuses: un article sur le peintre Cézanne dans le Quartier latin et un sur le poète Saint-Denys Garneau dans le SainteMarie (30 oct. 1945). Cinq polémiques: dans Combat avec Pierre Gélinas (décembre 1946), dans The Standard avec Eric MacLean (mai 1947), dans Notre temps avec Hyacinthe-Marie Robillard (décembre 1947), dans le Canada avec Agnès Lefort (novembre 1948) et dans le Petit Journal avec Pierre Saint-Germain (novembre-décembre 1949). Sans oublier deux lettres aux éditeurs André Laurendeau du Devoir (septembre 1948) et T. Damien Bouchard du Clairon (octobre 1948) à propos du renvoi de Paul-Émile Borduas.

Dans le Haut-parleur il est question surtout de ce qui touche le plus aux intérêts de Gauvreau: Picasso, Cocteau, Dada, Giguère, Pirandello et même Lili Saint-Cyr, la célèbre effeuilleuse de l'époque!

Dès le 14 janvier 1951, Gauvreau établit, comme position critique, le pluralisme:

accepter comme naturelle et bienfaisante la multiplicité des différences.

Les racistes et nationalistes sont des semeurs de haine ${ }^{6}$.

Ce pluralisme, il tente de l'associer à une impossible objectivité qu'il décrit assez naïvement à son correspondant d'alors, Jean-Claude Dussault, en des termes où on notera cependant le retour des mots réalité et objet pour désigner l'œuvre (façon de dire empruntée, comme on verra plus loin, au vocabulaire des peintres automatistes).

Celui-là ne pourra jamais comprendre mon comportement de critique, qui n'aura pas tenu compte du dédoublement extraordinaire dont je possède la puissance en moi. Le critique qui juge, ce n'est pas l'artiste qui produit. Je suis parvenu à pouvoir envisager toute réalité ou tout objet - y compris moi-même - comme si ma personne véritablement n'existait pas, comme si j'étais l'observateur bénévole d'une autre planète.

Quand je regarde un objet de quelque pays ou de quelque époque, ce ne sont pas des affinités avec ma propre ṕroduction que je cherche (ni que je fuis, la question ne se pose pas). Je suis exclusivement à l'affút de l'authentique justifiable?

Que faut-il penser de cette théorie du dédoublement dont il est ici question? Tentative d'objectivité ? II faudrait surtout dire tentative de ne pas confondre l'objet perçu et l'objet produit, le toi et le moi.

Pour le reste, on aura remarqué aussi une conception très "optique" de la critique où reviennent des expressions comme envisager, observateur, regarder à l'affût. Conception très décisive en même temps avec des mots comme: juge, justifiable. La tendance à s'ériger en juge autant que celle de se poser en observateur resteront une marque constante de cet écrivain formé par un peintre exigeant. Même le mot "cyclope", employé dans un de ses derniers titres, révèle la constance de cette critique du regard.

Gauvreau, s'il visa jamais à l'objectivité, ne cherche pas à s'y maintenir longtemps. II est personnellement impliqué, tantôt enferré par Jacques 
Ferron pour avoir dit de trop belles choses de Paul Toupin ${ }^{8}$, tantôt engagé dans une vengeance personnelle contre les jésuites ${ }^{9}$ où le règlement de comptes l'emporte sur les comptes rendus. II fait même la publicité d'une exposition qu'il aide à organiser, "les Rebelles» (7 avril et 21 juillet), donne des commentaires élogieux de la revue que fonde un groupe de ses amis et qui ne paraîtra que trois fois, Place publique ( 7 avril ), présente son exposition “les Étapes du vivant (26 mai), commente un des livres qu'il admire le plus, Égrégores de Pierre Mabille ( 9 juin), parle des productions de ses amis Jean-Claude Dussault, Roland Giguère, Guy Viau et André Goulet. Gauvreau apparaît alors comme un critique de plus en plus épris du parti pris idéologique.

Parti pris baroque, aussi; état d'esprit plutôt que style, qu'il décrit ainsi à propos de la pièce Magruhilne et la vie écrite en 1952:

Magruhilne et la vie est une tragédie baroque. C'est-à-dire que le texte est exempt de vraisemblance, et qu'il est surtout exempt de comique. Le texte est quelque chose de grave, de sensible; il ne se départit pas, cependant, de la sorte de sérénité bien spéciale qui est coutumière à l'imagination en liberté 10 .

Ce néo-baroque de Gaurreau désigne donc la totale liberté, la débauche d'imagination propre à ce mouvement qui fit concurrence aux classiques ${ }^{11}$.

Après un temps de réclusion, Gauvreau collabore à l'Autorité du peuple, un journal tout aussi engagé que ceux de Bouchard, où le poète parle de Fernand Leduc (mai 1953), du Salon du printemps (mars et avril 1954) et de son exposition "La Matière chante" (avril, mai et juin 1954) avec le même parti pris que dans le Haut-parleur. Son admiration pour Fernand Leduc, en particulier, l'amène à présenter des textes à Arts et pensée (juillet-août 1954) et au Journal musical canadien (mai 1955); mais ce ne sont plus là collaborations régulières et la critique de Gauvreau prend un autre souffle. Elle tend en particulier à mettre de l'ordre dans le passé - déjà - des automatistes, comme en témoignent les articles de Situations sur le dixième anniversaire du Refus global (février 1959) ou les articles sur Jean-Paul Martino (la Réforme, mai 1957), Micheline Beauchemin (Culture vivante, 1966), et Denis Vanier (Pornographic Delicatessen, 1968). Avec, comme on voit, de longs moments de silence.

\section{L'Emmerdeur}

Parallèle aux comptes rendus de Gauvreau, il y a les grands textes polémiques où la violence verbale ne démentit pas le parti pris de ses comptes rendus non plus que l'agressivité de sa poésie. Le premier grand texte polémique est "Cézanne, la vérité et les vipères de bon ton", en 1945. Gauvreau s'y décrit avec vigueur sous les traits inquiétants qu'on lui connaîtra jusqu'à la mort et qu'il se complaît à accuser:

II y a les emmerdeurs, ceux qui sont agités par la grandeur de la vie et la vitalité de leur esprit, ceux qui ne peuvent pas se résigner à voir 
leurs frères dans l'égocentrisme et la sécheresse lépreuse sans vouloir leur faire une part dans l'insatiable noblesse de ce qu'ils cherchent et trouvent. Les emmerdeurs prennent figure de dénonciateurs, de remords pour les êtres stationnaires, puisqu'ils accomplissent ce que, eux, n'ont pas eu le courage d'accomplir, puisqu'ils bravent l'incompréhension des pygmées et des pédants pour offrir à tous ceux qui ont faim d'enthousiasme et de vérité ce que la vie leur a enseigné être bon ${ }^{12}$.

Ce texte, de 1945, porte lourd le poids du critique qu'il décrit longtemps plus tard dans les mêmes termes. Mais, cette fois, ce sera dans la bouche des amis de son héros, et non pas dans celle des adversaires de l' "égrégore" automatiste, que les reproches seront placés. On reproche à Yvirnig ses textes polémiques:

COCHEBENNE - Ah! merde de merde! Ça devient franchement intolérable...

IVULKA - OUi... il a manqué une belle occasion de se faire oublier $[\ldots]$

CÓCHEBENNE - Tu es fou. Yvirnig, donc tu as tort en tout.

IVULKA - Les fous n'ont qu'à nous foutre la paix, ils n'ont qu'à prendre leur trou et à se faire oublier.

COCHEBENNE - Que la leçon te serve, cher Yvirnig! Nous avons soupé de tes élucubrations. Laisse-nous tranquilles! Tais-toi et n'écris plus une ligne! [...] Ah! Et puis merde à tous ceux qui sont incapables de porter un jugement par eux-mêmes ${ }^{13}$ !

Les emmerdeurs, ce sont les critiques comme Gauvreau, qui ne lâchent jamais: ne pas se résigner, dénoncer, lever les remords, braver, tels sont leurs agirs.

Les textes polémiques de Gauvreau sont construits de façon telle que le lecteur passe brusquement d'une phrase lyrique à une phrase didactique, des mots violents aux mots abstraits. Qu'on en juge par cet extrait d'une réponse au Père Robillard où Gauvreau manie d'abord I'ironie pour abattre son opposant:

Par aucune noirâtre manigancerie avec Lucifer devrai-je usurper des secrets d'alchimie pour parvenir à cette réalité simple et saine. Le mot: automatisme, malgré ce qu'en ont pu dire ou penser certains extasiés prudents qui croient sans doute à Merlin l'Enchanteur, ne s'encombre d'aucun pouvoir hypnotique ou magique et les hommes de bonne volonté pourront lire cet article sans craindre de perfides envoûtements sataniques ${ }^{14}$.

Gauvreau prend ensuite la défense du arigoureux et incorruptible Borduas ${ }^{15}$, pour donner enfin une explication de l'automatisme et l'insérer dans l'histoire des arts et lettres:

Avec la découverte de la photographie qui décrétait irrémissiblement la mort du figuratif coincidait presque une autre découverte scientifique, celle de l'inconscient par le docteur Sigmund Freud. Le monde extérieur visible ayant picturalement épuisé ses données, voilà que maintenant le monde intérieur, intime, de l'homme fournissait un panorama aussi riche de possibilités, de matières [...]

Automatisme [sic] s'applique donc à toute forme d'art où l'acte de création tire son point d'origine initiale non pas dans le monde exté- 
rieur immédiatement connu mais dans le monde intérieur de l'homme (qui est en fait le monde extérieur médiatement connu mais transformé dans l'inconscient et associé de façon particulière). L'intérieur de l'homme faisant partie intégrante de la nature, la discipline automatiste n'a donc pas une origine plus extraterrestre qu'aucune autre discipline d'art ${ }^{16}$.

C'est la douche écossaise, le traitement par l'alternance du chaud et du froid. La critique baroque, en somme, qu'on retrouve aussi dans d'autres articles du même genre. Qu'on songe à «Aragonie et surrationnel» ou “A propos de miroir déformant». Ces textes polémiques ont toujours le même caractère, celui de la Charge qu'il eut le courage et la lucidité de décrire dans la Charge de l'orignal épormyable ${ }^{17}$. Charge contre l'autre, de celui qui fonce contre l'obstacle, et c'est le premier temps; charge contre soi, de celui qui prend sur soi le fardeau de la preuve, et c'est le deuxième temps.

\section{Le théoricien}

Les textes didactiques de Gauvreau sont nombreux. Interprétation de l'histoire, comme "l'Épopée automatiste vue par un cyclope" où l'histoire est portée près de la légende et interprétée d'un regard subjectif. Analyse d'œuvres poétiques comme "les Affinités surréalistes de Roland Giguère" où l'auteur situe alternativement ses positions de poète et celles du poète dont il fait la critique.

“L'Épopée» est un texte majeur à lire pour comprendre Gauvreau. La subjectivité volontaire que l'œil unique du Cyclope veut signifier (dixit Gauvreau), n'empêche pas le regard de saisir le réel dans toute sa richesse. Même que, dans Les Oranges sont vertes, pièce à laquelle Gauvreau travaillait alors en même temps que Jappements à la lune, on a l'impression que le critique est le personnage central du groupe et que toute l'activité de l'égrégore automatiste dépend de lui. Cet égocentrisme n'apparaît pas dans “l'Épopée» où, au contraire, Paul-Émile Borduas et les Sagittaires d'abord, les Automatistes ensuite, évoluent dans une perspective sociohistorique beaucoup moins réductrice. (Je ne veux pas dire que le texte des Oranges soit réducteur, mais que la perspective serait réductrice si on voulait faire de cette pièce une reconstitution objective de l'époque, ce qu'elle n'est pas et ne prétend pas être.) Dans “l'Épopée», les grands manifestes que sont Refus global et Projections libérantes sont situés dans des perspectives tout aussi larges et audacieuses que précises et parfois anecdotiques.

«Les Affinités surréalistes de Roland Giguère» est le deuxième texte important de Gauvreau sur son ami, peintre et graveur. Le premier s'intitulait "Roland Giguère poète du Nouveau-monde ${ }^{18}$ " où déjà apparaissaient les jugements essentiels de Gauvreau sur Giguère:

Roland Giguère est un poète.

$\mathrm{Y}$ a-t-il dix Canadiens français de qui on puisse écrire cette phrase? 
Roland Giguère est le plus Européen de nos poètes vivants. Je veux dire par là qu'il est le moins baroque [...]

$\mathrm{Ce}$ n'est pas chez Giguère que l'on retrouvera une analogie de la fougue Milléresque, barbare, vociférante, volcanique. Giguère n'est pas touffu et énorme; Giguère est mesuré [...]

Son instinct de l'harmonie musicale est le plus subtil que j'aie connu sur le sol canadien.

Ces qualités d'une pensée achevée distinguent sensiblement Giguère des brutes magnifiques qui honorent habituellement nos arts frustes et sains (je pense à Paul-Marie Lapointe principalement) [...]

Lapointe, Giguère, cela fait deux.

II est clair, dans ces lignes, que Gauvreau se range parmi les "baroques canadiens" avec Lapointe derrière Arthur Miller, alors que Giguère lui apparaît plus près de Paul Éluard, de René Char et d'André Breton poète ${ }^{19}$.

La conférence du 12 février 1970 au Musée d'art contemporain explique la dichotomie entre «Européens " et "Canadiens » que Gauvreau utilise. La dichotomie se situe entre le baroque et la mesure:

Je vois le surréalisme actuel avec l'œil de Borduas, Giguère le voit avec l'œil de Pellan [...] Moi qui suis un baroque fruste, j'ai toujours envié à Giguère [...] le délié de sa phrase, la minutieuse fragilité resserrée de ses images, sa concision qui transmet plus d'électricité extasiante que n'importe quelle prolixité ${ }^{20}$.

La distinction entre le baroque et la mesure se dédouble chez Gauvreau, d'une distinction que la même conférence rappelle et qui apparaît très tôt dans la critique du poète: l'abstraction figurative et l'abstraction non figurative.

II n'y a pas d'abstraction proprement dite sans modèle extérieur. Giguère n'est ni un abstrait géométrique ni un abstrait tout court; ses sources d'enthousiasme, d'invention sont intérieures et cela ne s'était jamais vu avant l'abstraction ou pendant celle-ci. Par contre, il y a le non-figuratif sans modèle extérieur; le célèbre automatisme est de cette veine ${ }^{21}$.

Cette distinction était plus explicite dans une autre conférence prononcée au Musée d'art contemporain le 21 juin 1967, texte de type plutôt historique qui a pu servir de premier jet à «l'Épopée automatiste vue par un cyclope ". Dans la conférence de 1967, Gauvreau montre que les distinctions qu'il fait parmi les différents types d'abstractions sont précisément celles que Paul-Émile Borduas faisait dans Refus global. Il cite alors des extraits de "Commentaires sur des mots courants" qui étaient conçus pour la peinture mais qui, repris dans la conférence de Gauvreau, apparaissent clairement valables en littérature. On voit même que ces "Commentaires" adjoints au manifeste surrationnel ont servi de théorie littéraire à Gauvreau.

Après avoir souligné que nous devons aux surréalistes la révélation de "l'importance morale de l'acte non préconçu", Borduas définit comme suit l'automatisme psychique [...]: "A cause de la mémoire utilisée, l'intérêt se porte davantage sur le sujet traité (idée, similitude, image, association imprévue d'objets, relation mentale) que sur le sujet réel (objet plastique, propre aux relations sensibles de la matière 
employée)." Quant à l'automatisme surrationnel, Borduas le décrìt comme suit: "Écriture plastique non préconçe. Une forme en appelle une autre jusqu'au sentiment de l'unité, ou de l'impossibilité d'aller plus loin sans destruction. /En cours d'exécution, aucune attention n'est portée au contenu. L'assurance qu'il est fatalement lié au contenant justifie cette liberté./ Complète indépendance morale vis-à-vis l'objet produit. II est laissé intact, repris en partie ou détruit selon le sentiment qu'il déclenche (quasi impossibilité de reprise partielle). Tentative d'une prise de conscience plastique au cours de l'écriture (plus exactement peut-être a un état de veille»). Désir de comprendre le contenu une fois l'objet terminé 22 ."

On a reconnu, dans les textes cités de Borduas, un élément essentiel de l'argumentation de Gauvreau, argumentation qu'il rappelle dans sa conférence, toujours dans le but de se mieux situer, lui, baroque, entre les deux autres abstractions (psychique et géométrique). II tient à se distinguer de l'abstraction psychique (surréaliste) du groupe de Pellan, et de l'abstraction géométrique des néo-plasticiens québécois qui font école à Montréal après les départs de Borduas et de Pellan (1953). De ces derniers surtout, il dit:

Les automatistes étaient tous convaincus que l'abstraction baroque non-régularisée était une forme d'expression complètement ultérieure à celle de l'abstraction régularisée de Mondrian. Ne perdons pas de vue qu'un écart de vingt-cinq ans sépare l'abstraction géométrique de l'abstraction baroque 23 .

Terminons sur ces mots-clés de la pensée critique de Gauvreau une partie de la présente étude pour ouvrir une autre série de textes qui, eux, sont presque entièrement inédits. Car le texte principal sur la critique de Gauvreau, celui dont il sera question ici plus en détail, est Dix-sept lettres à un fantôme.

Les Dix-sept lettres s'échelonnent du 30 décembre 1949 au 10 mai 1950. Elles portent sur toutes sortes de sujets, au gré, parfois, des démarches ou des réflexions du récipiendaire.

La première parle longuement du désir et de la beauté convulsive, s'inspirant des idées de Breton dans Château étoilé:

La révolution de «l'art", comme toutes les révolutions, d'ailleurs, n'est pas un but en soi ; la révolution est une conséquence, certes inéluctable, mais elle n'est pas plus qu'un effet.

Le but de l'activité artistique, comme de toutes les démarches conséquentes d'ailleurs, est d'extérioriser, de concrétiser le désir [...]

Le seul professeur qui ne sera pas néfaste pour ses élèves est celui qui basera toujours ses critiques sur les qualités sensibles objectives.

Peu importe l'aspect d'une œuvre, peu importe la discipline intellectuelle qui l'a permise, peu importe le degré d'évolution intellectuelle de son auteur; seule importe la "beauté convulsive" - d'une nature strictement sensible - qui aura été inscrite objectivement dans la matière ${ }^{24}$.

Cette lettre parle donc du poème comme object concret. La suivante revient sur les mots qui disent l'empirisme de la «poésie où le langage, au 
lieu de demeurer un signe conventionnel pour évoquer des états préalablement connus, devient organiquement une réalité sensible autonome et absolument concrète ${ }^{25}$. Gauvreau apporte aussi une distinction importante pour comprendre et sa critique et sa production, la distinction entre le but et la conséquence, distinction entre ce qu'une œuvre veut dire (son intention) et ce qu'elle dit (son effet) :

Je crois qu'on aurait tort de supposer à “l'intention» - «la bonne intention" - un pouvoir qu'elle n'a jamais eu et qu'elle n'aura jamais.

II ne suffit pas de caresser le but intentionnel d'aider les autres, de faire avancer la connaissance, pour parvenir à réaliser cette intention. De même il y a des foules de conséquences extrêmement excellentes qui sont entièrement imprévisibles ${ }^{26}$.

J'insiste pour vous mettre en garde contre l'illusion de "comprendre" une poésie avec laquelle le seul contact réalisé a été un contact analytique $[\ldots]$.

La connaissance poétique d'un objet ne réside pas du tout dans la capacité de savoir comment sa naissance fonctionne. Elle réside dans la capacité inénarrable de VIBRER à chacune de ses fluctuations, à chacune de ses épaisseurs et minceurs, à chacun de ses contrastes, à chacune de ses explorations, internes ou externes ${ }^{27}$.

On notera au passage le mot "exploration" qui prendra chez Gauvreau un sens particulier qu'on retrouvera tantôt. Entretemps, notons que l'auteur renchérit encore sur l'objectivité, sur la matérialité de l'œuvre d'art dans ces lettres que nous citons abondamment parce qu'elles sont d'une richesse critique exceptionnelle pour l'époque et sont pourtant demeurées inédites. Gauvreau répond, par exemple, sans le savoir, à ceux qui se poseront plus tard à son égard le problème art-folie autant que tout autre problème d'interprétation de l'œuvre par la vie de l'auteur:

S'il vous plaît, ne confondez pas deux réalités bien séparées que tout le monde confond généralement: d'abord la manière dont l'œuvre prend naissance - Et ensuite la réalité propre et définitive de cette ouvre.

Une fois le cordon ombilical rompu, l'enfant est un tout complet et indépendant de sa mère. On peut en dire autant de l'objet d'art.

On a vu souvent des hommes - doués d'une libido généreuse mais affligés d'un égo bien exécrable - méconnaître eux-mêmes leurs propres productions [...]

Il faut en déduire qu'il existe deux séries de problèmes indépendants: les problèmes subjectifs du créateur qui produit - problèmes qui n'intéressent que lui-même et ses confrères. Et ensuite, les problèmes objectifs des cuvres - problèmes universels, collectifs, qui intéressent la connaissance et la critique.

Une cuvre dont on ignorerait tout de l'auteur, dont on ignorerait même l'origine, serait-elle moins valable? Bien sûr que non.

Jugeons donc un objet sur ses qualités sensibles intrinsèques c'est par ce seul côté qu'il peut avoir une valeur sociale et qu'il peut influer sur l'évolution et qu'il peut faire jouir et enrichir ${ }^{28}$. 
Productions, produire, objets, efficacité, travailleurs: ces mots de la nouvelle critique, Gauvreau et Borduas les utilisaient déjà en janvier 1950! Gauvreau, comme Borduas, prend pourtant ses distances avec les matérialistes autant qu'avec les idéalistes:

Sur le sujet de l'idéalisme et du matérialisme, je voudrais vous expliquer que le terme «idéaliste " qualifie ou qualifiait généralement ceux qui croient que l'état d'une société peut être transformé par une simple prise de conscience et par la persuasion; les «matérialistes", par contre, seraient ceux qui croient qu'un simple effort de pensée est impuissant à modifier quoi que ce soit et que l'évolution obéit à des lois matérielles extérieures aux idées.

It me semble que la solution du matérialisme et de l'idéalisme serait le réalisme anthropomorphique [...]

Pour ma part, je pense que toute volonté individuelle est incapable de transformer quoi que ce soit de profond - du moins si elle s'exerce à l'encontre du déterminisme collectif. Je crois aussi que les périodes d'évolution sont favorisées et rendues possibles par des prises de conscience individuelles (au sujet de ce déterminisme) prises de conscience rendues générales au moment propice.

II serait idéaliste de croire que l'apport surréaliste pourrait devenir social avant que la sensibilité collective fût suffisamment disponible pour le recevoir.

D'autre part, sans la prise de conscience surréaliste, les périodes de carnages pourraient être suivies inutilement de longues périodes d'errement et de dépression sans solution ${ }^{29}$.

La solution surréaliste lui paraît donc distincte de l'approche idéaliste; distincte aussi de l'approche matérialiste des staliniens. Gauvreau rejette par exemple péremptoirement le jdanovisme:

Le jdanovisme a été et demeure (jusqu'à nouvel ordre) une doctrine officielle de l'URSS en esthétique. Pourquoi cette doctrine est-elle ce qu'elle est? Elle est ce qu'elle est parce que I'URSS - depuis quelque 35 ans - est un capitalisme d'état façonné par des penseurs médiocres (dont le séminariste Djougachvili), établi et maintenu au profit d'une nouvelle classe de privilégiés: une classe de bureaucrates, de bureaucrates éduqués en petits-bourgeois et dont toutes les conceptions et toutes les aspirations sont celles des petitsbourgeois de l'univers pré-soviétique ${ }^{30}$.

Chacun de nous comprendra la nécessité d'instaurer un système économique propre à rendre impossible l'exploitation financière (dont nous sommes nous-mêmes les victimes) - mais chaque révolutionnaire devra comprendre qu'aucun intellectuel, à aucun prix, ne pourra tolérer un régime qui impliquera le dirigisme moral [...]

Du reste, le totalitarisme économique n'impliquerait nullement en soi les tracassières régimentations intellectuelles ${ }^{31}$.

Solution surréaliste, donc abondamment inspirée des textes de Breton comme Rupture inaugurale, et que Gauvreau concentre auto'sr de la notion de monisme athée ou de réalisme anthropomorphique:

Par monisme, j'entends la conviction qu'un seul principe constitutif préside à tous les phénomènes concevables dans l'univers. 
Pour le moniste, l'esprit et la matière, en dernière analyse, c'est la même chose. Seuls les aspects de l'éternel devenir changent pour la lucidité anthropomorphique ${ }^{32}$.

La notion de réalisme anthropomorphique, qui tend à résoudre les contradictions d'une adhésion surréaliste à la pensée matérialiste (surtout dans la mesure où le surréalisme réclame la liberté totale et anarchique pour l'artiste), Gauvreau la fait dériver nettement de la notion de réalisme ouvert que Breton utilise pour définir le surréalisme: "réalisme ouvert ou surréalisme qui entraîne la ruine de l'édifice cartésien-kantien et bouleverse de fond en comble la sensibilité ${ }^{33}$ ". Le même texte de Breton, “Limites non-frontières du surréalisme" que les automatistes avaient découvert grâce à Gilles Hénault, recourait à une distinction entre contenu manifeste et contenu latent d'une ceuvre, considérant que le réalisme socialiste réduisait l'cuvre au seul contenu manifeste. Gauvreau prend à son compte cette distinction qu'il décrit en ces termes à Dussault:

Le contenu manifeste serait les proportions intrinsèques de l'objet: la progression et l'entremêlement des rythmes. Ces chocs verbaux, les particularités imagènes, les relations [...] Le contenu latent serait les préoccupations inconscientes [...] dictant tel choix d'épithètes, tel accent, tel amalgame, telle cassure, telle disjonction, etc. ${ }^{34}$.

$\mathrm{Ce}$ «réaliste anthropomorphique" qu'est Gauvreau doit parfois, en critique, inventer de son crû. Comme par exemple quand il parle d'images et qu'il définit ainsi l'image: “l'association ou la mise en confrontation de n'importe quels éléments verbaux: syllabe, mot abstrait, mot concret, lettre, son, etc. ${ }^{35}$.». II distingue quatre grands types d'images auxquels il donne des noms à sa façon.

La première est l'image rythmique, c'est-à-dire une onomatopée ou une percussion qui ne serait pas strictement sonore (comme le retour périodique d'une couleur ou d'un symbole). La deuxième est l'image mémorante, c'est-à-dire la substance imagène que sont les métaphores et métonymies ${ }^{36}$. La troisième est l'image transfigurante, c'est-à-dire un procédé linguistique analogue à la surimpression photographique, à la totémisation verbale pratiquée par les surréalistes (cf. «Mattatoucantharide ${ }^{37}$ ou encore au collage verbal ${ }^{38}$. La quatrième est l'image exploréenne ${ }^{39}$, c'est-à-dire une espèce de sarabande inconsciente, de débauche verbale:

On parle d'image exploréenne lorsque les éléments constitutifs des nouveaux éléments singuliers ne sont plus immédiatement décelables, par une opération analytique. Je dirais aussi qu'il y a image exploréenne lorsque la situation présente de la psychanalyse ne permet pas à cette science - à moins peut-être d'une opération laborieuse dont il n'existe pas encore d'exemple - de découvrir en l'objet poétique le contenu latent ${ }^{40}$.

On peut croire aujourd'hui que cette opération existe, au contraire, si on se réfère aux études parues sur le logogriphe obsessionnel ${ }^{41}$. Mais là n'est point la question; et pour clore ce dernier volet de la pensée critique de Gauvreau, il faut principalement rappeler l'influence de l'Esthé- 
tique de Hegel sur Breton ${ }^{42}$ et l'influence de Breton sur Gauvreau et sur tous les automatistes:

Je vous recommande fermement - à titre d'accostage - deux livres d'André Breton qui circulent actuellement à Montréal. Ce sont Nadja et L'Amour fou.

Breton [...] utilise constamment la projection.

Même dans ses écrits théoriques, qui sont nombreux, Breton s'exprime par projection. II transmet ainsi des nuances, inacessibles aux plus dialecticiens.

Les esprits rationnels ne peuvent souffrir Breton; les esprits surrationnels, par contre, lui sont d'une reconnaissance inépuisable.

Tâchez de vous procurer les manifestes du surréalisme rédigés par Breton. Ce sont des cargos de dynamite dont l'explosion définitive n'a pas encore eu lieu ${ }^{43}$.

On peut ainsi croire que les écrits théoriques de Gauvreau, comme ceux de Breton, ont été rédigés dans le même mouvement de projection que ses œuvres créatrices. Ils correspondent comme elles à la projection d'un désir.

On semble alors justifié de parler, à propos de Gauvreau, de critique baroque comme il a lui-même parlé de baroque à propos de sa production "surrationnelle". Baroque au sens "composite" du terme, puisque Gauvreau et les automatistes ont pris leur bien où ils ont cru bon le faire; les repères critiques de Gauvreau sont empruntés par le biais des surréalistes, tantôt à Freud, tantôt à Marx. Baroque au sens "profus " et "généreux" du terme, puisque l'œuvre de Gauvreau est une des plus abondantes et des plus diversifiées de notre littérature, et que ces cuvres sont toujours animées d'un souffle puissant qui emporte tout sur son passage. Baroque au sens «libertaire" du terme, puisque la critique de Gauvreau est complètement anarchique par rapport aux canons de l'époque au Québec. Qui donc, hormis Gauvreau, se permettait, en 1950, de parler de Gisèle Prassinos ou d'Henri Pichette sur les tribunes québécoises? Qui donc osait parler de poésie en termes d'objet, de production?

Le sujet abordé ici n'est sans doute pas épuisé par ces quelques pages, surtout sur la question des rapports avec Breton et le freudomarxisme. Mais il est à espérer que la richesse du matériel critique de Gauvreau aura été suffisamment indiquée pour que non seulement on sache qu'elle existe mais qu'on s'y penche plus intensément encore.

André G. Bourassa Université d'Ottawa

1. Extrait d'un livre à paraître aux éditions l'Étincelle à l'automne.

2. Claude Gauvreau, Les oranges sont vertes, in CEuvres créatrices complètes, p. 1420. On aura noté le chiasme "phrases de poésie... syllabe de critique". C'est plutôt la poésie (chez Gauvreau) que désignerait le mot "syllabe ". 
3. Id., Etal mixte, photo de Kéro, Montréal, éditions d'Orphée, 1968; recueil mis sur le marché en juin 1977 avec permission des éditions Parti pris (202 exemplaires sur 1000), 71 pages.

4. Id., CEuvres créatrices complètes, coll. du chien d'or, Montréal, éditions Parti pris, 1977 (copyright 1971), 1503 pages.

5. Id., «Barroques [sic] canadiens dans les Pays-Bas», le Haut-parleur, 3 février 1951, p. 2.

6. Id., "Question de propagande ", le Haut-parleur, 14 janvier 1951, p. 5.

7. Id., Dix-sept lettres à un fantôme, 2 mai 1950, p. 13.

8. Jacques Ferron, «Lettre ouverte à Claude Gauvreau sur les vaches» (sur les affinités Toupin-Montherlant), le Haut-parleur, 3 mars 1951; "Lettre de Toupin à Gauvreau ", ibid., 17 mars 1951; «/e Choix de Paul Toupin " (sur certains traits de fascisme de Montherlant et de Toupin), ibid., 7 avril 1951.

9. Claude Gauvreau, "Robert Gadouas a le courage de tenir tête aux censeurs", le Haut-parleur, 21 avril 1951; "Ostracisme jésuitique - les coupures invraisemblables commandées par le Gesù » (28 avril) ; “Au pays des Jésuites - Lili Saint-Cyr et les délectations moroses" (14 juillet); «Au-delà de l'immondice jésuitique - André Goulet dit Goulo" (22 décembre).

10. Id., CEuvres créatrices complètes, op. cit., p. 270.

11. L'expression “baroque" est assez fréquente autour de Gauvreau pour que le correspondant des Dix-sept lettres à un fantôme, Jean-Claude Dussault, l'ait intégrée deux fois de suite dans des poèmes parus dans le Haut-parleur, "Nuit d'été " et «Sacrificial " (21 janvier 1951).

12. Claude Gauvreau, "Cézanne, la vérité et les vipères de bon ton ", le Quartier latin, 9 février 1945, p. 5.

13. Id., Les Oranges sont vertes, in CEuvres créatrices complèțes, op. cit., p. 14201421.

14. Id., "L'Automatisme ne vient pas de chez Hadès», 1'e partie, Notre temps, 6 décembre 1947, p. 3.

15. Ibid.

16. Ibid., $2^{8}$ partie, 13 décembre 1947, p. 6. N.B. La page du journal est datée du 6 , par erreur.

17. Cf. la polémique autour de la pièce qui devient en elle-même une nouvelle charge. Claude Gauvreau, "la Mort de I'Orignal épormyable", la Presse, 16 mai 1970, p. 49; suivi de Monique Du Plantie, Jacques Crête et Albert G. Paquette, “Une nouvelle charge épormyable", la Presse, 23 mai 1970, p. 41.

18. Claude Gauvreau, in le Haut-parleur, 28 juillet 1951, p. 5.

19. Voir là-dessus Gauvreau, "Quelques poètes inconnus", le Haut-parleur, 30 juin 1951, p. 5 et 7 . II y est question de Lautréamont, Germain Nouveau, Arthur Cravan, Jacques Vaché, Guillaume Apollinaire, Max Jacob, Blaise Cendrars, Reverdy, Tristan Tzara, Francis Picabia, Paui Éluard, André Breton, Antonin Artaud, Gisèle Prassinos, Aimé Césaire et Henri Pichette. Noter que l'article est de 1951 !

20. Claude Gauvreau, «les Affinités surréalistes de Roland Giguère", in Études littéraires, vol. $5 \mathrm{n}^{\circ} 3$, décembre 1972, p. 502 et 505 .

21. Ibid., p. 509.

22. Id., in Quebec Underground 1962-1972, t. 1, Montréal, les Éditions Médiart, 1973 , p. 66. "Commentaires sur des mots courants" ajoutait les noms de Lautréamont après "liberté " et de Robert Elie après «veille".

23. Ibid.

24. Id., Dix-sept lettres à un fantôme, 30 décembre 1949, p. 24-26; inédit.

25. Ibid., 7 janvier 1950, p. 3 ; inédit.

26. Ibid., p. 9 ; inédit.

27. Ibid., 19 avril 1950, p. 9 ; inédit.

28. Ibid., 7 janvier 1950, p. 10; inédit.

29. Ibid., 8 février 1950, p. 26-27 ; inédit.

30. Id., "Aragonie et surrationnel ", la Revue socialiste, $n^{\circ}$ 5, printemps 1961, p. 67. 
31. Id., Dix-sept lettres à un fantôme, 26 avril 1950, p. 27 ; inédit.

32. Ibid., 10 mai 1950, p. 23 ; inédit. Voir, sur le monisme, Gérard Durozoi et Bernard Lecherbonnier, le Surréalisme, théories, thèmes, techniques, Paris, Larousse, “Thèmes et textes", 1972, p. 84-87.

33. André Breton, "Limites non-frontières du surréalisme", in la Clé des champs, Paris, Pauvert, 1967, p. 18.

34. Claude Gauvreau, Dix-sept lettres à un fantôme, 1 ब̄r février 1950, p. 12-13; inédit.

35. Ibid., p. 8 ; inédit.

36. Ibid., $\mathcal{1}^{\text {er }}$ avril 1950, la Barre du jour, nos 17-20, cf. p. $349-353$ pour l'image rythmique, p. 353 pour l'image mémorante.

37. Marcel Jean, Histoire de la peinture surrealiste, Paris, Seuil, 1959, p. 124.

38. Cf. Max Ernst, Écritures, Paris, NRF, "Le Point du jour", 1970, p. 262.

39. Le qualificatif s'explique peut-être par cet extrait d'une lettre à Dussault où Gauvreau explique en termes d'exploration la thèse par laquelle Borduas [«Projections libérantes», p. 26] refusait l'«intention»: “Les travailleurs surrationnels ne peuvent pas renier leur découverte capitale, celle qui ouvrira les portes les plus vastes sur l'inexploré: la conséquence est plus importante que le but. " (Dix-sept lettres à un fantôme, 30 mars 1950, p. 22-23)

40. Claude Gauvreau, Dix-sept lettres à un fantôme, in la Barre du jour, nos 17-20, p. 357-361.

41. René Major rappelle un genre d'analyse qu'il fait remonter à Freud: “Des mots prononcés sont toujours les vestiges de mots vus ou entendus. Les rêves nous en fournissent souvent des exemples. Mais il faut avouer qu'à cet égard les sources du langage de la vie vigile peuvent être plus difficiles à retracer que celles du langage onirique, puisque dans le rêve les images verbales, cédant le plus souvent la place aux images visuelles, sont plus circonscrites [...].

"Certaines formules, authentiques libellés d'obsessions, se donnent d'emblée comme énigmes à déchiffrer. Freud en fournit un bel exemple dans "l'Homme aux rats»: une incantation en forme d'acrostiche, de son patient Lorentz, "Glejisamen», exprime consciemment un vœu magique: "puisse Lorentz être heureux maintenant et toujours, amen ". Le décriptage de cet acrostiche révèle qu'il cache l'anagramme du nom de la dame vénérée, Gisela, qu'il mettait ainsi en contact, dans sa formule, avec le sperme (samen). Trouvant sa place dans l'expression condensée, l'amen final venait ponctuer sa prière."

Major analyse ensuite les expressions d'un patient: «Ah! l'inceste!» («Alain, cesse !»); «ma marie-jeanne» (être le mari de Jeanne; marijuana; Marie Janet); "Sanzit-Montront " (sans zizi, mon rond)." (René Major, "le Logogriphe obsessionnel ", Interprétation, janvier-mars 1968, vol. 2, $n^{\circ} 1$. Les deux paragraphes cités sont des pages 5 et 6. .)

42. André Breton, "Situation surréaliste de l'objet", in Manifestes du surréalisme, Paris, Pauvert, 1962, p. 309-310.

43. Claude Gauvreau, Dix-sept lettres à un fantôme, op. cit., 22 mars 1950, p. 10; inédit. 